\title{
Knocking down Stard3 decreases adipogenesis with decreased mitochondrial ROS in 3T3-L1 cells
}

Xiaoqian Zhou ${ }^{\mathrm{a} \dagger}$, Huanqing $\mathrm{Gao}^{\mathrm{b} \dagger}$, Yinfeng Guo ${ }^{\mathrm{c}}$,Yaxi Chen ${ }^{\mathrm{a}, *}$, Xiong Z. Ruan ${ }^{\mathrm{ad}, \mathrm{e}^{*}}$

Xiaoqian Zhou:

${ }^{\mathrm{a} C e n t r e ~ f o r ~ L i p i d ~ R e s e a r c h ~ \& ~ K e y ~ L a b o r a t o r y ~ o f ~ M o l e c u l a r ~ B i o l o g y ~ f o r ~ I n f e c t i o u s ~}$ Diseases (Ministry of Education), Institute for Viral Hepatitis, Department of Infectious

Diseases, the Second Affiliated Hospital, Chongqing Medical University, 400016 Chongqing, China. email:zhouzhai123154026@163.com

Huanqing Gao

${ }^{b}$ Departments of Biology,Southern University of Science and Technology, Shenzhen,China. email:gaohq@sustc.edu.cn

Yinfeng Guo

${ }^{\mathrm{c} I n s t i t u t e}$ of Nephrology, Zhong Da Hospital, Southeast University School of Medicine, Nanjing, Jiangsu 210009, China. xiaoguoyf@163.com

Yaxi Chen

${ }^{\mathrm{a} C e n t r e ~ f o r ~ L i p i d ~ R e s e a r c h ~ \& ~ K e y ~ L a b o r a t o r y ~ o f ~ M o l e c u l a r ~ B i o l o g y ~ f o r ~ I n f e c t i o u s ~}$ Diseases (Ministry of Education), Institute for Viral Hepatitis, Department of Infectious

Diseases, the Second Affiliated Hospital, Chongqing Medical University, 400016 Chongqing, China email: chenyaxi@cqmu.edu.cn

Xiong Z. Ruan

${ }^{\mathrm{a} C e n t r e ~ f o r ~ L i p i d ~ R e s e a r c h ~ \& ~ K e y ~ L a b o r a t o r y ~ o f ~ M o l e c u l a r ~ B i o l o g y ~ f o r ~ I n f e c t i o u s ~}$ Diseases (Ministry of Education), Institute for Viral Hepatitis, Department of Infectious Diseases, the Second Affiliated Hospital, Chongqing Medical University, 400016 Chongqing, China. email: x.ruan@ucl.ac.uk

${ }^{\mathrm{d}}$ The Collaborative Innovation Center for Diagnosis and Treatment of Infectious Diseases (CCID), Zhejiang University, 310058 Hangzhou China, 
eJohn Moorhead Research Laboratory, Centre for Nephrology, University College London Medical School, Royal Free Campus, University College London, London NW3 2PF, United Kingdom.

* Corresponding authors Addresses: Centre for Lipid Research \& Key Laboratory of Molecular Biology for Infectious Diseases (Ministry of Education), Institute for Viral Hepatitis, Department of Infectious Diseases, the Second Affiliated Hospital, Chongqing Medical University, 400016 Chongqing, China. E-mail addresses: chenyaxi@cqmu.edu.cn (Y.Chen), x.ruan@ucl.ac.uk (X.Z. Ruan)

$\dagger$ The authors contribute equally as joint first authors. 


\title{
Knocking down Stard3 decreases adipogenesis with decreased mitochondrial ROS in 3T3-L1 cells
}

\begin{abstract}
:
Start domain-containing protein 3 (Stard3) plays roles in intracellular cholesterol distribution, however, the role of Stard3 in the adipogenesis of 3T3-L1 preadipocytes remains unclear. We demonstrated that Stard3 expression was significantly increased during the adipogenesis of 3T3-L1 preadipocytes, accompanied by an increase of mitochondrial Reactive oxygen species (ROS). Stard3 knocking-down inhibited 3T3L1 preadipocyte adipogenesis with decreased mitochondrial ROS levels, while ROS inducer rescued the stard3 silencing 3T3 cells with increased ROS. Moreover, Stard3 silencing reduced the expression of peroxisome proliferator-activated receptor- $\gamma$ (PPAR $\gamma)$ and CCAAT/enhancer binding protein (C/EBP) $\alpha$ in 3T3- L1 cells. In conclusion, Stard3 enhanced the adipogenesis of preadipocytes by enhancement of cholesterol redistribution to the mitochondrial, increasing mitochondrial ROS production. These results suggest that Stard3 is an essential factor for the 3T3-L1 cells' differentiation.
\end{abstract}

\section{Keywords}

Start domain-containing protein 3, Cholesterol, adipogenesis

\section{Introduction}

Adipogenic differentiation is defined as the process of changing from preadipocytes to 
mature adipocytes. Since this process is closely related to the fat accumulation and the formation of obesity, which is a risk factor for Type 2 diabetes mellitus, cardiovascular disease, and metabolic syndrome[1], the mechanisms of adipocyte differentiation is imperative to study. Adipogenesis is a multistep process that is regulated by a complex of transcriptional factors such as the peroxisome proliferator-activated receptor- $\gamma$ (PPAR $\gamma$ ) family and CCAAT/enhancer binding protein (C/EBP) including C/EBP a , $\mathrm{C} / \mathrm{EBP} \beta[2 ; 3]$. PPAR $\gamma$ and $\mathrm{C} / \mathrm{EBP}$ a are key factors in the regulation of the expression of genes leading to adipocyte development. Reactive oxygen species (ROS) can also promote adipogenesis, which is demonstrated by different studies[4; $5 ; 6]$. Lee et al. found that the ROS promoted adipogenic differentiation by acceleration of mitotic clonal expansion[4]. Carriere reported firstly that inhibition of mitochondrial ROS production could prevent 3T3-L1 proliferation[7]. Tormos also showed that ROS promoted adipocyte differentiation by increasing mitochondrial metabolism, which was accompanied with the upregulation of $\mathrm{C} / \mathrm{EBP} \alpha$ and $\operatorname{PPAR} \gamma[8]$.

The differentiation of preadipocytes is characterized by increased triglyceride production. Studies have found that fat cell cholesterol levels are closely related to cell size[9]. With increasing amounts of triglycerides, the amount and distribution of cholesterol is increased correspondingly in the fat cells. Increased storage of triglyceride in fat cells can also lead to its intracellular cholesterol redistribution[10]. Adipogenesis was also reported to be regulated by adipocyte cholesterol amount[11; 12]. Stanton et al. demonstrated the role of low-density lipoprotein cholesterol in 
stimulating the differentiation of 3T3-L1 in vitro[11]. Although the above studies illustrated the importance of cholesterol for the adipogenesis in 3T3-L1 cells, the role of cholesterol in adipogenesis is conflicting. Le lay's research on 3T3-L1 adipocytes showed no visible morphologic changes when differentiating in a cholesterol-deprived medium in the presence of an inhibitor of sterol synthesis [13], implying that intracellular cholesterol location, rather than cholesterol levels, could be a key factor for adipogenesis.So it is imperative to investigate the role of cholesterol location in 3T3-L1 cells adipogenesis.

Start domain-containing protein 3 (Stard3), also called MLN64, is expressed in different tissues [14] and it has a C terminal domain called C-terminal steroidogenic acute regulatory protein (StAR)-related lipid transfer(START). Stard3 is one of the subfamilies of the steroidogenic acute regulatory-related (StAR) lipid transfer protein, which has a high affinity for cholesterol. Charman et al. found that Stard3 regulates cholesterol transport to mitochondria, particularly when cholesterol homeostasis is altered such as in Niemann-Pick Disease[15]. With cholesterol accumulating on the mitochondrial membrane, excessive ROS is produced [16]. The differentiation of adipocytes is associated with ROS, and cholesterol plays a major role in the process of adipogenesis. However, there is no study on the role of Stard3 in adipocyte differentiation.

In the current study, we reported that Stard3 protein increased after the induction of 
differentiation in 3T3-L1 cells with increased cholesterol redistribution to the mitochondria and mitochondrial ROS, while Stard3 silencing suppressed adipogenesis with decreased mitochondrial ROS by decreasing the delivery of cholesterol to the mitochondria. This suggests that Stard3 mediated the cholesterol redistribution involved in adipogenesis.

\section{Materials and methods}

\subsection{Cell culture}

3T3-L1, a murine preadipocyte cell line (a gift from Prof Fiona M. Watt in Kings College London) was seeded in $6 \mathrm{~cm}^{2}$ dishes and maintained in a high glucose concentration (4.5 g/L D-glucose) Dulbecco's Modified Eagle Medium (Sigma, UK), supplemented with $10 \%$ (vol/vol) heat-inactivated calf serum, at $37{ }^{\circ} \mathrm{C}$ and $5 \% \mathrm{CO}_{2}$.

\subsection{Cell differentiation}

Preadipocytes were seeded in $6 \mathrm{~cm}^{2}$ dishes until confluence occurred. Two days postconfluence (designed as day 0), the medium was removed and replaced with DMEM with $10 \%$ fetal serum (Labtech, UK), $0.5 \mathrm{mM}$ 3-isobutyl-1-methylxanthine (IBMX), 5ug/ml insulin(Sigma, USA) and 1uM dexamethasone (Sigma, USA) for 2 days. After 2 days (day 2), the cells were cultured in a growth medium with $5 \mathrm{ug} / \mathrm{ml}$ insulin for another 2 days. Then, the medium was swapped with growth medium and renewed every other day from day 4 until day 8 . 


\subsection{Oil red-O staining}

Oil Red-O (ORO) staining was taken to quantify the lipid accumulation. Eight days after induction of differentiation, cells were fixed with formaldehyde (4\% in PBS) for 30 min at room temperature after being washed with phosphate buffered saline (PBS) three times. Cells were then washed twice with PBS and stained with ORO dye (3:2, $0.6 \%$ ORO dye in water) for $30 \mathrm{~min}$. ORO was extracted with $100 \%$ isopropanol, and the absorbance was determined at $510 \mathrm{~nm}$.

\subsection{Small interfering RNA}

3T3-L1 preadipocytes were transfected with small interfering RNA (siRNA) with Lipofectamine RNAiMAX (Invitrogen, UK), using siRNA targeting murine Stard3 or a non-targeting control (Invitrogen, UK) at a final concentration of $75 \mathrm{nM}$ for 24 hours before induction of differentiation (Day-1). siRNA sequences were listed in Supplementary Table S1.

\subsection{Quantitative real-time PCR}

Total RNA was isolated from cells using TRizol Reagent (Invitrogen, UK) according to manufacturers' instructions. Two micrograms of total RNA were converted to firststrand complementary DNA in 20ul reactions using a cDNA synthesis kit (Applied Biosystems, UK). Real-time reverse transcription polymerase chain reaction (PCR) was performed in a real-time PCR machine, using SYBR Green dye (Lab science, UK). To normalize the expression data, $\beta$-actin was used as an internal control gene, and the data 
was analyzed using the $\Delta \mathrm{Ct}$ method. The primer sequences used in this study were shown in Table S1.

\subsection{Protein isolation and Western blot analysis}

Total proteins were extracted from 3T3-L1 cells with RIPA buffer supplied with a protease inhibitor cocktail (Sigma, USA). Identical amounts of sample proteins were separated by SDS-PAGE. After being transferred to PVDF membranes, the membranes were blocked for one hour with 5\% BSA. Then the membranes were incubated with primary antibodies (anti-PPAR $\gamma$, anti-FABP4, anti-cebp $\alpha$ from Cell Signaling Technology, USA, anti-Stard 3 from Abcam, UK) overnight at $4^{\circ} \mathrm{C}$. The antibody for $\beta$ actin from Sigma (USA) was used as a loading control. After that, the membrane was incubated with corresponding horseradish peroxidase-labelled secondary antibody for 1 hour. Finally, detection procedures were performed with Immobilon Western Chemiluminescent HRP Substrate (Millipore, USA). The membrane was developed with ECL. Then, the membrane was stripped by stripping buffer (Sigma, USA) and reprobed for actin as loading control. Band intensities were measured by Image $\mathbf{J}$ software $1.46(\mathrm{NIH})$.

\subsection{Mitochondrial ROS measurement}

3T3-L1 cells were plated on the disk and treated by corresponding treatment. Cells were incubated with $5 \mu \mathrm{M}$ MitoSox (Invitrogen, USA) for 30 minutes after rinsing with PBS. After the final wash in PBS, the images acquired using a Zeiss laser scanning confocal 
microscope. The level of mitosox fluorescence was measured at an excitation wavelength of $510 \mathrm{~nm}$ and an emission wavelength of $580 \mathrm{~nm}$ by a microplate reader (BioTek, USA) and was normalized by blue fluorescence stained with Hoechst (SigmaAldrich, USA). The results were expressed as a percentage of the control. The whole process was performed in the dark.

\subsection{Statistical analysis}

Values are presented as means \pm standard deviation $(\mathrm{SD})$, with significance $(\mathrm{P}<0.05)$ determined by an unpaired test. Data is presented as the mean \pm SD of three independent experiments.

\section{Results}

3.1. Stard3 and mitochondrial ROS increased in the process of the adipogenic differentiation

To confirm whether Stard3 is involved in the adipogenic process, we determined the Stard3 expression in different time courses of Stard3 expression during the 3T3-L1 adipocyte differentiation into mature adipocytes. In normally differentiated 3T3-L1 cells, the protein levels of Stard3 were increased after adipogenic induction, accompanied by increased expression of transcription factors in adipogenesis, such as PPAR $\gamma$, cebp $\alpha$ and FABP4 in the progress of adipogenesis (Figure 1A,B). The mRNA levels of Stard3 and the transcriptional factors presented a similar change corresponding with protein levels (Fig.1C). To confirm whether mitochondrial ROS is involved in the 
adipogenic process, we also determined the mitochondrial ROS level at different times during the 3T3-L1 adipocyte differentiation into mature adipocytes (Fig 1.D) and demonstrated that mitochondrial ROS was also increased during the adipogenic process

\subsection{Stard3 silencing decreased lipid accumulation}

To assess the effect of Stard3 on the adipogenesis of 3T3-L1 preadipocytes, Stard3 was knocked down with siRNA interference. ORO staining and quantitative assays revealed that the silencing of Stard3 resulted in a decreased neural lipid deposition in 3T3-L1 cells (Figure. 2).

3.3. Stard3 silencing reduced the adipogenic gene expression and mitochondrial ROS Stard3 silencing significantly reduced the protein levels of Stard3, PPAR $\gamma, \operatorname{cebp} \alpha$, FABP4 on Day 0, Day 4 and Day 8 when compared with control cells (Figure 3A, B ). Stard3 silencing reduced the mRNA levels of cebp $\alpha$, PPAR $\gamma$, FABP4 significantly. The mRNA levels of Stard3 and adipogenic factors presented a similar change corresponding with protein levels. (Figure3C). The mitochondrial ROS presented a similar change corresponding with the protein levels of Stard3 (Figure3D).

\subsection{TBHP rescued the Stard3 silencing 3T3-L1 cells with increased ROS}

The mitochondrial ROS increased in 3T3-L1 cells when treated with Tert-Butyl hydroperoxide (TBHP) in 3T3-L1 cells (Figure 4A,B). The increased expression of cebp $\alpha$ and PPAR $\gamma$ by Stard3 silencing in 3T3-L1 cells was rescued when treated with 
TBHP (Figure 4C,D).

\section{Discussion}

Adipogenesis is characterized as a process in which preadipocytes proliferate and differentiate from precursor cells into mature adipocytes. The process involves changes in morphology, hormone sensitivity and gene expression [17]. Adipocytes express transcription factors, which can influence adipogenesis directly. During the process of adipocyte differentiation, C/EBP $\alpha$ and PPAR $\gamma$ are essential[18; 19]. ROS induced C/EBP $\beta$ and increased PPAR $\gamma$ in early preadipocyte differentiation [20; 21]. In the current study, we also showed during the process of adipocyte differentiation the adipogenic genes were upregulated, and ROS generation was increased.Mitochondria need to keep a stable mitochondrial membrane potential (MMP) and redox transitions to preserve function and integrity. In different pathological conditions, excess mitochondrial cholesterol results in redundant mitochondrial ROS production[22]. A previous study has shown the depolarization of mitochondrial membrane was induced by ROS generation in response to accumulation of mitochondrial cholesterol, hence changing the rigidity of the mitochondrial membrane. The overexpression of Stard3 decreases MMP and increases mitochondrial ROS production[16]. These results suggest that cholesterol may regulate adipogenesis by the ROS pathway.

Adipogenesis is a process by which a new adipocyte formed from a preadipocyte with increased lipid (mainly triglycerides) synthesis. However, cholesterol may also play an 
important regulatory role in adipogenesis. Interestingly, Le lay's research on 3T3-L1 adipocytes showed no visible morphologic changes when differentiating in a cholesterol-deprived medium in the presence of an inhibitor of sterol synthesis [13], suggesting that cholesterol intracellular location, rather than cholesterol levels, might be a key factor for the regulation of adipocyte differentiation. Cholesterol is synthesized in the endoplasmic reticulum(ER) and trafficked to different cellular organelles such as the mitochondria or plasma membrane(PM)[15]. Cholesterol, as a basic membrane component, maintains the integrity of sub-organelles and vesicular trafficking[23]. Cholesterol also regulates the membrane lipid order, which modulates membrane permeability. When excessive cholesterol accumulated on the mitochondrial membrane, the transport of L-Glutathione (GSH) to the mitochondria was impaired, which led to less mitochondrial ROS depletion [16]. During the trafficking progress, Stard3 could provide a binding site for cholesterol trafficking[24]. Elisa Balboa showed the upregulation of Stard3 could transport cholesterol to mitochondria, which induced cholesterol accumulation on mitochondria[16].

Furthermore, our current study demonstrated that Stard3 directly regulates adipogenesis. We found that silencing stard3 was able to inhibit adipocyte differentiation and lipid accumulation, tested by ORO staining of cytosol lipids. The potential mechanism could be the inhibition of cholesterol trafficking from the organelles to the mitochondria, decreasing the mitochondrial ROS. The inhibition of mitochondrial ROS results in a decreased expression of transcriptional factors PPAR $\gamma, \mathrm{CEBP} \alpha$ and FABP4. 
Taken together, Stard3, an important molecule component of cholesterol homeostasis, plays an important role in the progress of adipocyte differentiation and could be a new target for modulating adipogenesis.

\section{Acknowledgements}

Not applicable.

\section{Funding}

This research was supported by the National Natural Science Foundation of China key

Program, No. 81390354 and 81570517,31571210).

\section{References}

[1] J.M. Chan, E.B. Rimm, G.A. Colditz, et al., Obesity, fat distribution, and weight gain as risk factors for clinical diabetes in men, DIABETES CARE 17 (1994)961-9.

[2] A.G. Cristancho, M.A. Lazar, Forming functional fat: a growing understanding of adipocyte differentiation, Nat Rev Mol Cell Biol 12 (2011)722-34.

[3] S.R. Farmer, Transcriptional control of adipocyte formation, CELL METAB 4 (2006)263-73.

[4] H. Lee, Y.J. Lee, H. Choi, et al., Reactive Oxygen Species Facilitate Adipocyte Differentiation by Accelerating Mitotic Clonal Expansion, J BIOL CHEM 284 (2009)10601-10609.

[5] W. Wang, Y. Zhang, W. Lu, et al., Mitochondrial Reactive Oxygen Species Regulate Adipocyte Differentiation of Mesenchymal Stem Cells in Hematopoietic Stress Induced by Arabinosylcytosine, PLOS ONE 10 (2015)e0120629.

[6] F. Atashi, A. Modarressi, M.S. Pepper, The role of reactive oxygen species in mesenchymal stem cell adipogenic and osteogenic differentiation: a review, STEM CELLS DEV 24 (2015)1150-63.

[7] A.F.Y.R. Carriere, Inhibition of preadipocyte proliferation by mitochondrial reactive oxygen species, FEBS LETT (2003)163 - 167.

[8] K.V.A.E. Tormos, E. Anso, R.B. Hamanaka, et al., Mitochondrial complex III ROS regulate adipocyte differentiation, 2011, pp. 537 - 544.

[9] B.R. Krause, A.D. Hartman, Adipose tissue and cholesterol metabolism, J LIPID RES 25 (1984)97110 .

[10] M. Guerre-Millo, P. Guesnet, C. Guichard, et al., Alteration in membrane lipid order and composition in metabolically hyperactive fatty rat adipocytes, LIPIDS 29 (1994)205-9. 
[11] L.A. Stanton, M. van de Venter, D. Litthauer, et al., Effect of lipoproteins on the differentiation of 3T3-L1 and human preadipocytes in cell culture, Comp Biochem Physiol B Biochem Mol Biol 116 (1997)65-73.

[12] R. Masella, R. Vari, M. D'Archivio, et al., Oxidised LDL modulate adipogenesis in 3T3-L1 preadipocytes by affecting the balance between cell proliferation and differentiation, FEBS LETT 580 (2006)2421-9.

[13] S. Le Lay, S. Krief, C. Farnier, et al., Cholesterol, a Cell Size-dependent Signal That Regulates Glucose Metabolism and Gene Expression in Adipocytes, J BIOL CHEM 276 (2001)16904-16910.

[14] H. Watari, F. Arakane, C. Moog-Lutz, et al., MLN64 contains a domain with homology to the steroidogenic acute regulatory protein (StAR) that stimulates steroidogenesis, Proc Natl Acad Sci U S A 94 (1997)8462-7.

[15] M. Charman, B.E. Kennedy, N. Osborne, et al., MLN64 mediates egress of cholesterol from endosomes to mitochondria in the absence of functional Niemann-Pick Type C1 protein, The Journal of Lipid Research 51 (2010)1023-1034.

[16] E. Balboa, J. Castro, M. Pinochet, et al., MLN64 induces mitochondrial dysfunction associated with increased mitochondrial cholesterol content, REDOX BIOL 12 (2017)274-284.

[17] H.J. Cho, J. Park, H.W. Lee, et al., Regulation of adipocyte differentiation and insulin action with rapamycin, Biochem Biophys Res Commun 321 (2004)942-8.

[18] P. Tontonoz, B.M. Spiegelman, Fat and beyond: the diverse biology of PPARgamma, ANNU REV BIOCHEM 77 (2008)289-312.

[19] M.I. Lefterova, M.A. Lazar, New developments in adipogenesis, Trends Endocrinol Metab 20 (2009)107-14.

[20] Y. Hou, P. Xue, Y. Bai, et al., Nuclear factor erythroid-derived factor 2-related factor 2 regulates transcription of CCAAT/enhancer-binding protein beta during adipogenesis, Free Radic Biol Med 52 (2012)462-72.

[21] P. Tontonoz, E. Hu, B.M. Spiegelman, Stimulation of adipogenesis in fibroblasts by PPAR gamma 2, a lipid-activated transcription factor, CELL 79 (1994)1147-56.

[22] A. Boveris, B. Chance, The mitochondrial generation of hydrogen peroxide. General properties and effect of hyperbaric oxygen, BIOCHEM J 134 (1973)707-16.

[23] K. Simons, E. Ikonen, How cells handle cholesterol, SCIENCE 290 (2000)1721-6.

[24] J.B. Pinto, A. Graham, The role of endosomal cholesterol trafficking protein, StAR-related lipid transfer domain 3 (StarD3/MLN64), in BRIN-BD11 insulinoma cells, PROTEIN CELL 7 (2016)833838. 
Fig1.Expression of Stard3 was increased during 3T3-L1 cells adipogenesis

A. Adipogenesis of 3T3-L1 cells was induced with MDI. The protein levels of Stard3, cebp $\alpha$, FABP4 and PPAR $\gamma$ were compared at different time-points by immunoblotting. Actin was used as loading controls. B. Quantitative data of immunoblots. C. mRNA levels at time points of differentiation of 3T3-L1 preadipocyte (D0, D4 and D8 represented 0 day, 4 days and 8 days after induction of differentiation, respectively ). D. Mitochondrial ROS level was increased during 3T3-L1 cells adipogenesis. ${ }^{*} \mathrm{p}<0.05$ and $* * \mathrm{p}<0.01$ compared with D0 .

Fig. 2. Silencing of Stard3 blunted the differentiation of 3T3-L1 preadipocyte.

Images of differentiated 3T3-L1 cells stained with Oil Red O after treatment with siStard3 or scrambled siRNA on 8 days of induction of differentiation. Lipid accumulation was quantified by extracting Oil Red O. (siStard3: cells treated with siStard3; siNC: cells treated with non-targeting siRNA. $* * \mathrm{P}<0.01$ compared with siNC).

Fig. 3. siRNA-mediated silencing of Stard3 3T3-L1 inhibits the preadipocytes differentiation

A. siStard3 or non-target siRNA treatment on the protein expression of Stard3, PPAR $\gamma$, cebp $\alpha$, FABP4 in 3T3-L1 preadipocyte B.Quantitative data of immunoblots C. siStard3 or non-target siRNA treatment on the RNA level of Stard3, PPAR $\gamma$, cebp $\alpha$, FABP4 in 3T3L1 preadipocyte. D.Mitochondrial ROS level after Stard3 silencing on 3T3-L1 cells (*p < 0.05 and $* * p<0.01$ compared with non-target siRNA treatment at the corresponding time point).

Fig. 4. Stard3 silence induced 3T3-L1 preadipocytes differentiation inhibition was rescued when treated with TBHP.

A.Mitochondrial ROS level was increased when 3T3-L1 treated with sistard3 and TBHP.

B. Quantitative data of fluorescence. C. The expression of PPAR $\gamma$, cebp $\alpha$ in 3T3-L1 preadipocyte adipogenesis were rescued when 3T3-L1 cells knocked down by siStard3 was treated with TBHP. D. Quantitative data of immunoblots. *p $<0.05$ and compared with siNC. 
A

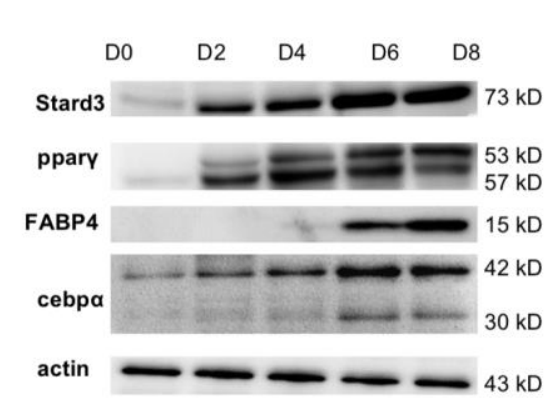

B

C
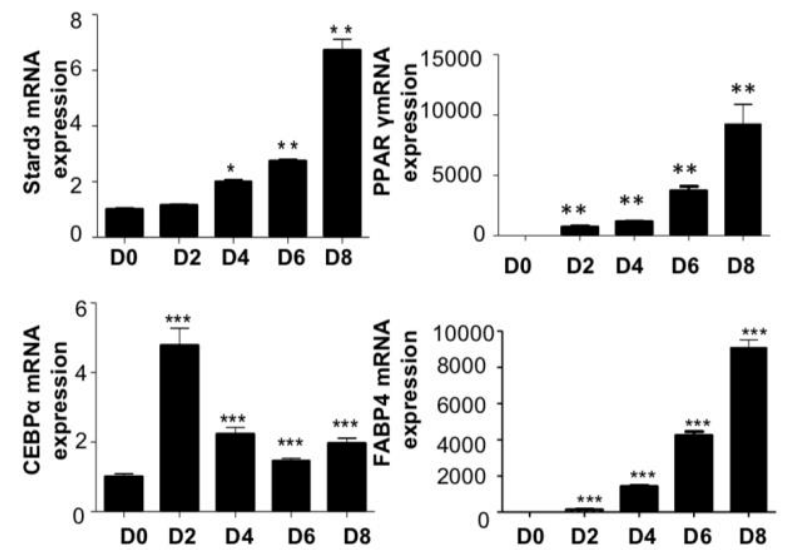

D

MitoSOX staining
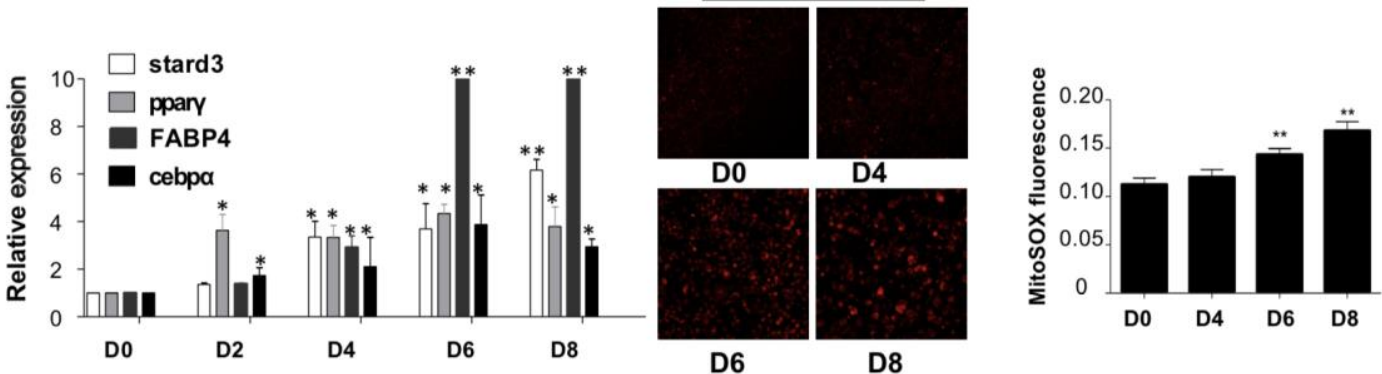

Fig1.Expression of Stard3 was increased during 3T3-L1 cells adipogenesis 

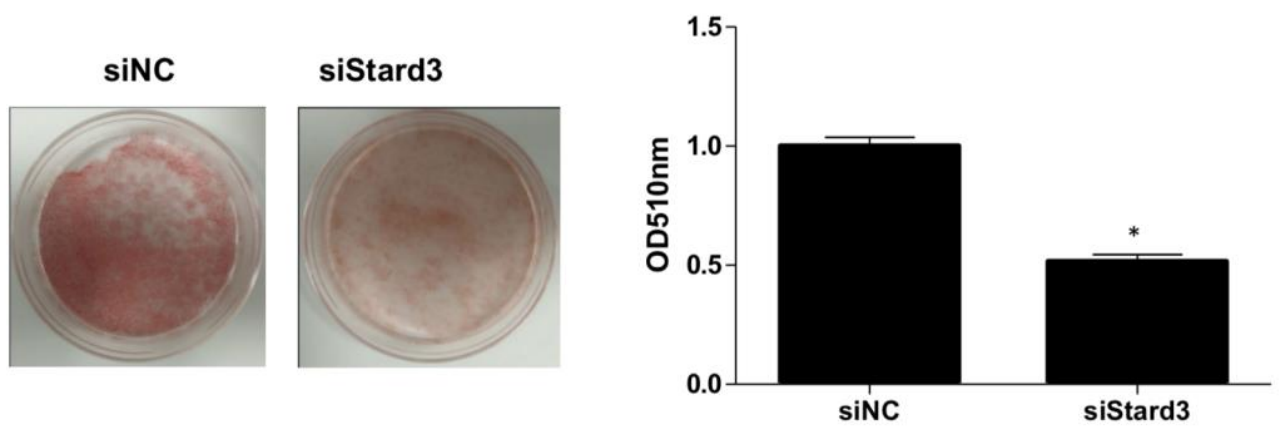

Fig. 2. Silencing of Stard3 blunted the differentiation of 3T3-L1 preadipocyte. 


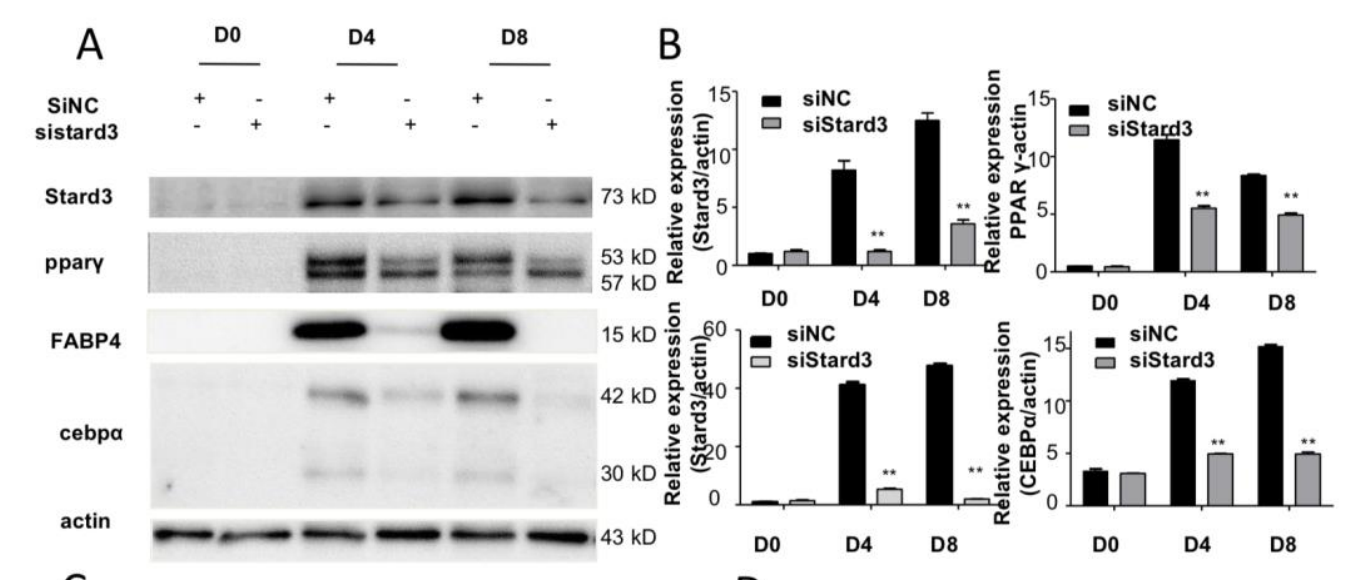

C
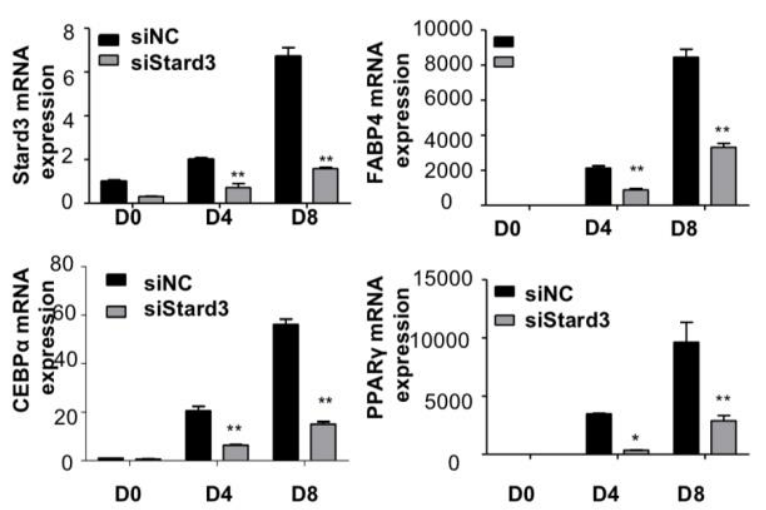

D

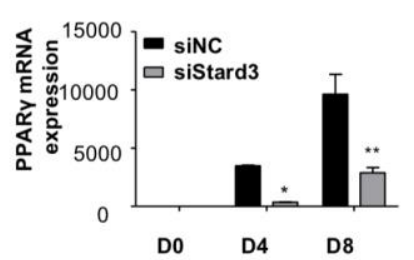

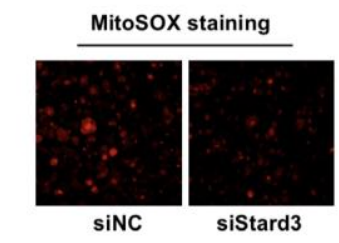

Fig. 3. siRNA-mediated silencing of Stard3 3T3-L1 inhibits the preadipocytes differentiation 
A

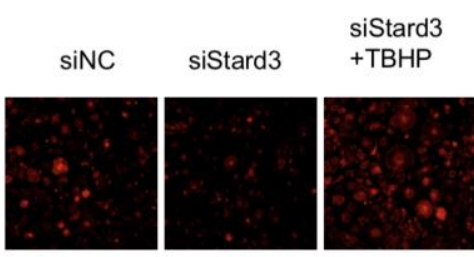

C

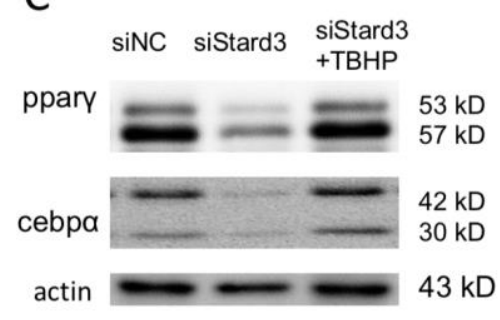

B
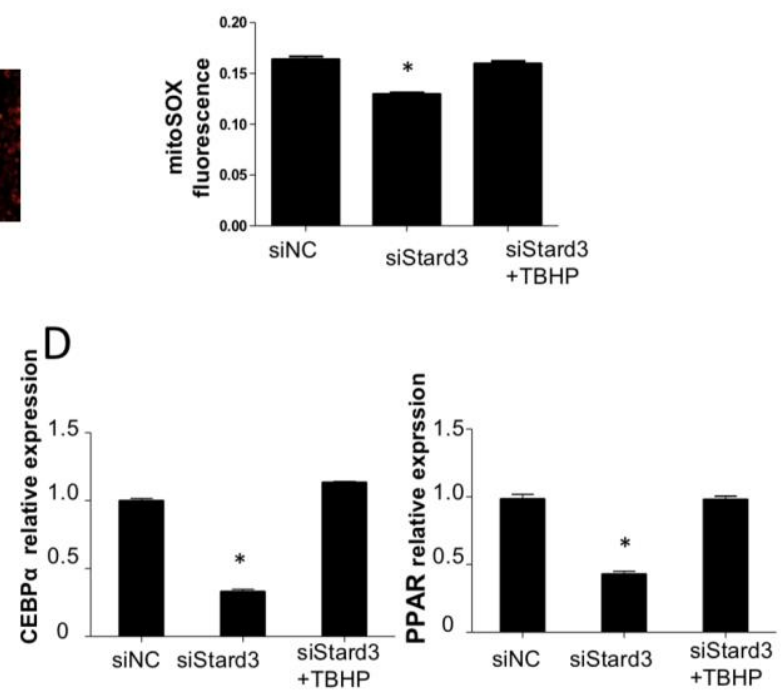

Fig. 4. Stard3 silence induced 3T3-L1 preadipocytes differentiation inhibition was rescuced when treated with TBHP. 